\title{
Correction to: Spontaneous rhythms in a harbor seal pup calls
}

\author{
Andrea Ravignani $i^{1,2,3^{*}}$
}

\section{Correction to: BMC Res Notes (2018) 11:3 https://doi.org/10.1186/s13104-017-3107-6}

Following publication of the original article [1], the author reported an error in the first sentence of the 'Sound recordings' section. The recordings mentioned in this sentence were performed 6 days later than written. In this Correction article the original and corrected version of the sentence are presented.

The incorrect sentence upon publication:

- On the twenty-first day from estimated birth, $10 \mathrm{~min}$ of vocalizations were recorded in air using a unidirectional microphone Sennheiser ME-66 (frequency response $40-20,000 \mathrm{~Hz} \pm 2.5 \mathrm{~dB}$; Sennheiser electronic GmbH\&Co. KG, Wedemark, Germany) [14].

The corrected sentence:

- On the twenty-seventh day from estimated birth, $10 \mathrm{~min}$ of vocalizations were recorded in air using a unidirectional microphone Sennheiser ME-66 (frequency response 40-20,000 $\mathrm{Hz} \pm 2.5 \mathrm{~dB}$; Sennheiser electronic GmbH\&Co. KG, Wedemark, Germany) [14].

The reference which is cited in the above sentence can be reviewed in the original article.

\begin{abstract}
Author details
${ }^{1}$ Research Department, Sealcentre Pieterburen, Hoofdstraat 94a, 9968 AG Pieterburen, The Netherlands. ${ }^{2}$ Artificial Intelligence Lab, Vrije Universiteit Brussel, Pleinlaan 2, 1050 Brussels, Belgium. ${ }^{3}$ Language and Cognition Department, Max Planck Institute for Psycholinguistics, Wundtlaan 1, 6525 XD Nijmegen, The Netherlands.
\end{abstract}

The original article can be found online at https://doi.org/10.1186/s1310 4-017-3107-6.

\section{Publisher's Note}

Springer Nature remains neutral with regard to jurisdictional claims in published maps and institutional affiliations.

Received: 21 May 2018 Accepted: 21 May 2018

Published online: 11 June 2018

\footnotetext{
Reference

1. Ravignani A. Spontaneous rhythms in a harbor seal pup calls. BMC Res Notes. 2018;11:3. https://doi.org/10.1186/s13104-017-3107-6.
} 